PART IV

HALO AND OLD DISC POPULATIONS 
A. RED VARIABLES AND EVOLUTION

ON THE GIANT BRANCHES 


\title{
RED VARIABLES OF THE OLD DISC AND \\ HALO POPULATIONS
}

\author{
M. W. FEAST
}

Radcliffe Observatory, Pretoria, South Africa

\begin{abstract}
This paper discusses the following topics: (1) Mira Variables in Globular Clusters; (2) infrared observations of Globular Cluster Variables; (3) carbon Stars in Magellanic Cloud Clusters; (4) the Se Variables and the superlithium rich Variables.

Red variables represent an important but little understood phase of stellar evolution. Primarily for this reason, they have attracted an increasing amount of attention in recent years. This paper is restricted to a discussion of three or four aspects of this topic which have been of particular interest to a group of us working at the Radcliffe Observatory.
\end{abstract}

\section{Mira Variables in Globular Clusters}

The subject of Mira variables in globular clusters was discussed fairly extensively at the Toronto Colloquium last year. Let me briefly summarise the present position. The Miras in the general field (stars with Me spectra) show a marked dependence of kinematic property on period (e.g. Feast, 1963; Feast et al., 1972). If we include with these variables, the Fe-Ke variables (the SRd variables of the General Catalogue of Variable Stars), we can form a kinematic sequence running from halo type objects with periods near 100 days down to disc objects of low velocity dispersion with periods of about 450 days (Feast, 1965; Preston, 1967). Examples of SRd Miras are found in some metal poor globular clusters; a result consistent with the halo kinematics of the SRd Miras in the general field. Seven globular clusters, all in the metal rich category, contain Miras with typical Me spectra. Until recently, there were only six periods known for stars in this group (three of these were in $47 \mathrm{Tuc}$ ). All these periods were near 200 days. This is consistent with the kinematics of field 200 day Miras which are intermediate between disc and extreme halo population types. Recent work (Lloyd Evans et al., to be published) has shown that two other clusters have Miras of longer period. NGC 6553 has a Mira of period 270 days and NGC 5927 one of 312 day period. The latter star is most likely a cluster member from its position in the centre of the cluster and its radial velocity is consistent with membership. There is some evidence (Feast, 1973; Lloyd Evans and Menzies, 1973) that these clusters may be more metal rich than the 47 Tuc type clusters which contain the 200 day variables (spectral type near giant tip, $(V-I)$ colours of variables). These results therefore tend to reinforce the idea that there is a fairly precise relation of period with chemical composition and/ or age for the Mira-SRd sequence. Most globular clusters containing Miras also contain smaller amplitude red variables which presumably show us how stars become pulsationally unstable as they approach the red giant tip and evolve with increasing amplitude and period, finally becoming Mira variables. 


\section{Infrared Photometry of Globular Cluster Variables}

A more detailed study of the sequence of events amongst variables at the red giant tip is desirable. As one contribution to such a study, Glass and Feast (1973) have been carrying out broad band infrared photometry in the 1.2-3.5 $\mu$ region $(J, H, K, L)$ of red giants and variables in 47 Tuc and $\omega$ Cen. The following is a summary of some of the results obtained.

The two colour $(J-H)-(H-K)$ plot yields some useful results. This type of plot is interesting because in it the population I luminosity classes I and III stars are well displaced from the black-body line. This is primarily due to a maximum in the stellar continuum in the $\mathrm{H}$ band $(1.6 \mu)$, caused by an opacity minimum in the stellar atmosphere at this wavelength. At longer wavelengths, $\mathrm{H}^{-}$free-free opacity dominates and $\mathrm{H}^{-}$bound-free at shorter wavelengths (Woolf et al., 1964, etc). It is found that the majority of the 47 Tuc and $\omega$ Cen stars studied fall near the position of the population I stars, well away from the black-body line. However, a striking feature of the globular cluster results is that the three 200 day Mira variables in 47 Tuc (V1, V2 and V3) lie much nearer the black-body line than the other stars. Fragmentary data on field Miras (Mendoza, 1967) indicate that their behaviour is similar to that of the 47 Tuc Miras. These results are presumably connected with the fact that, unlike other $\mathrm{M}$ stars, Miras show strong $\mathrm{H}_{2} \mathrm{O}$ absorption bands in the infrared which depress the $1.6 \mu$ continuum peak. At present, it is something of a mystery why Miras have strong $\mathrm{H}_{2} \mathrm{O}$ absorption bands and other $\mathrm{M}$ stars of similar temperature do not (Johnson et al., 1968). Abundance differences have been suggested or it could result from unspecified differences in atmospheric structure. In any event, the 47 Tuc results seem to show that the effect comes in quite abruptly for the Miras. In the $(J-H)$ $(H-K)$ plot, the SR-small amplitude red variables in 47 Tuc lie with normal M stars.

Although $M$ type stars have long been known in 47 Tuc, it was generally supposed that such stars would be confined to metal rich globular clusters. It was therefore quite a surprise when a few $M$ type variables with periods of about 100 days and amplitudes in the range 0.4 to 1.4 were found in the metal poor globular cluster $\omega$ Cen (Dickens et al., 1972). The $J, H, K, L$ photometry leads to some interesting results for these stars. It is found that the relation between the infrared colours and the spectral types as determined from TiO band strengths is the same as for field population I stars. Despite this, there are good reasons to suppose that these stars are, like other cluster members, metal poor, since they have large $(U-B)$ excesses $\left(\sim 0^{\mathrm{m}} \cdot 4\right)$ for their types and infrared colours. It has generally been assumed that, in giants of a given temperature, the TiO bands would be a sensitive indicator of metal abundance. On the contrary, our results show no dependence of TiO strength on metal abundance. A likely reason for this is that, in the spectral region where we study the TiO bands, $\mathrm{TiO}$ is itself a major source of opacity so that, to a first approximation, band strength is independent of metal abundance. Detailed calculations are needed to test this hypothesis. 
The infrared colours indicate that the normal red giant branch in $\omega$ Cen ends at $T_{\text {eff }} \sim 3800^{\circ}$. At this point, the stars have $\log L / L_{\odot}=3.23$ and their positions agree well with the first giant branch tip (onset of the helium flash) calculated for $Z=10^{-3}$ (Rood, 1972; Demarque and Mengel, 1973). This value of $Z$ is consistent with that estimated for $\omega$ Cen in other ways; $(U-B)$ excess, abundance analysis of Fehrenbach's star (Dickens and Powell, 1973). The $M$ type variable members lie at considerably cooler temperatures $\left(\sim 3100^{\circ}\right)$ but at about the same luminosity $\left(\log L / L_{\odot}=3.16\right)$. Since the helium flash occurs at a point on or close to the Hayashi line, we deduce that these variables are beyond the Hayashiline and presumably therefore in a stage of rapid evolution probably connected with mass loss.* It would obviously be of importance to decide whether these variables are on their first or second ascent of the giant branch. There is a strong temptation to believe that they are at the top of the giant branch for the second time. One may then maintain that these stars are showing the first signs of instability which leads to the ejection of a shell, the rapid evolution back across the HR diagram and the formation of a planetary nebula. Whilst this may indeed be the case, it appears to leave little place in the scheme of things for the two CH stars in $\omega$ Cen.** These carbon stars lie near the tip of the giant branch proper and an attractive suggestion would appear to be that they represent the tip of the second giant branch whilst the $M$ variables are in a rapid excursion to the right of the Hayashi line from the tip of the first giant branch. If the $M$ variables are indeed first giant branch stars, then they might well take care of the mass loss necessary to provide a spread of masses on the horizontal branch. A decision between the two possible alternatives for the $\mathbf{M}$ variables will evidently rest to a large extent on detailed calculations of stellar evolution at the helium flash.

\section{Some Problems of the Carbon-rich Red Variables}

To understand the evolutionary status of variable carbon stars (and carbon stars in general), we badly need to know their bolometric magnitude and temperatures. At the present time the temperature calibration seems to be particularly uncertain. Bessell and Youngbom (1972) have found that the temperatures of $N$ type stars derived from continuum scans in the region $7000-11000 \AA$ are lower than those derived from $(R-I)$ measures calibrated from $M$ stars $(2500$ to $2900 \mathrm{~K}$ rather than 3300 to $3500 \mathrm{~K}$ ). This is presumably due to the effects of strong $\mathrm{CN}$ absorption. However it is not certain that even Bessell and Youngbom's work refers to the true continuum. Evidently a great deal will need to be known about the infrared spectra before broad band colours can be properly interpreted.

An interesting problem in connection with carbon stars is the nature of some of the

\footnotetext{
* The results of Glass and Feast (1973) leave open the possibility that in 47 Tuc, the Mira variables may lie relatively close to the Hayashi track.

** A third carbon star has recently been reported in the region of $\omega$ Cen but this has been found to be a radial velocity non-member (Catchpole and Feast, 1973).
} 
very red stars in globular clusters in the Magellanic Clouds which distinguish these clusters from globular clusters in our own Galaxy. Several of these very red stars in SMC globular clusters have now been examined spectroscopically (Feast, 1973; Feast and Lloyd Evans, 1973). The reddest star in Kron $3(B-V=2.57)$ is a carbon star. There is at least one carbon star and probably more in NGC 419 and the small amplitude variable V8 in NGC 121 is a carbon star. No such very red carbon stars (variable or non-variable) are known in galactic globular clusters (the $\mathrm{CH}$ stars in $\omega$ Cen being much bluer). However recent work suggests that red carbon stars may be rather frequent in intermediate age clusters in the Galaxy, (see the discussion of Catchpole and Feast (1973)). Of the small number of known intermediate age clusters, three (NGC 7789, 2660 and 2477), all with ages near $1.5 \times 10^{9} \mathrm{yr}$, are now know to contain carbon stars. This result suggests that the occurrence of these stars is rather sensitive to age and gives some attraction to the hypothesis that the SMC globular clusters may be somewhat younger than typical galactic globular clusters. Gascoigne (1966) and others have indeed suggested on other grounds that Kron 3 and NGC 419 might be intermediate age clusters but the situation remains rather confused.

In discussing small amplitude carbon variables such as NGC $121 \mathrm{~V} 8$, it should be borne in mind that whilst none are known in galactic globular clusters, such stars may be present in the galactic halo population. The majority of known carbon $(\mathrm{CH})$ stars in the halo population have $(B-V)$ in the range of about 1.00 to 1.55 like the $\mathrm{CH}$ stars in $\omega$ Cen. However Table I lists two small range carbon stars with colours rather

TABLE I

\begin{tabular}{lllllllll}
\hline Star & $\begin{array}{l}\text { Period } \\
(\text { days })\end{array}$ & $\begin{array}{l}\text { Approx. } B \\
\text { amp. }\end{array}$ & $B-V$ & $\begin{array}{l}U \\
\left(\mathrm{~km} \mathrm{~s}^{-1}\right)\end{array}$ & $\begin{array}{l}V \\
\left(\mathrm{~km} \mathrm{~s}^{-1}\right)\end{array}$ & $\begin{array}{l}W \\
\left(\mathrm{~km} \mathrm{~s}^{-1}\right)\end{array}$ & $\varrho$ & $b$ \\
\hline NGC 121, V8 & 112 & 0.4 & $1.7-2.15$ & & & & & \\
TT CVn & Irr & 0.7 & $+1.85(\max )$ & -28 & -353 & -74 & -135 & +79.2 \\
V Ari & 77 & 1.0 & $+2.1(\max )$ & +107 & +19 & +345 & -176 & -45.7 \\
\hline
\end{tabular}

similar to NGC $121 \mathrm{~V} 8$. Also listed are the components $U, V, W$ of their space motions (Eggen, 1972) and their observed radial velocities and galactic latitudes. Both stars appear to be halo objects and presumably indicate that very red carbon stars are not entirely lacking in some component of this population.

\section{The Se Variables and the Super Lithium Rich Variables}

The Se variables are an intriguing and little understood group of stars. The limited data on their radial velocities suggest that they cover a wide range of ages since the majority show clearly the effects of differential galactic rotation (e.g. Feast 1963) whilst one Se variable is known with a radial velocity of $329 \mathrm{~km} \mathrm{~s}^{-1}$ w.r.t.l.s.r. (Catchpole and Feast, 1971) making it a halo object. The period of this latter star is 251 
days (Andrews, 1973) which does not clearly distinguish it from Se variables of the low velocity group.

The Se variables may be of importance in helping solve the problem of the superlithium rich stars. It has been suggested (Cameron and Fowler, 1971) that the superlithium rich stars occur as a result of a brief phase of stellar evolution involving extensive convection down to a helium burning shell. In addition it has been suggested that the lithium formed in this way is a major source of ${ }^{7} \mathrm{Li}$ in the universe (e.g. Truran and Cameron, 1971).

Keenan (1967) and Boesgaard (1970) have found the Se variable T Sgr to be superlithium rich with a lithium abundance as great or greater than the $\mathrm{T}$ Tauri stars. Recently, a survey has been carried of lithium in southern $S$ stars (Catchpole and Feast, to be published). Out of $169 \mathrm{~S}$ stars observed in this programme, only one is in the superlithium rich class. This is the Se variable RZ Sgr which is rather similar to T Sgr. Since our $169 \mathrm{~S}$ stars contain only $27 \mathrm{Se}$ stars, the frequency of superlithium rich Se stars may be quite high, possibly higher than amongst carbon stars despite the fact that for many years the only known superlithium rich stars were the carbon stars WZ Cas, WX Cyg and T Ara. T Ara was found in a survey of 50 carbon stars (Feast, 1953). Warner and Dean (1970) observed another 158 carbon stars without finding another superlithium rich star. Recently about 70 more carbon stars have been observed (mostly by Catchpole) without finding any superlithium rich stars.

Superlithium rich stars are apparently particularly frequent in the intermediate CS and SC classes with two superlithium rich objects in the small group of only 18 known stars, most of which are SR variables, (Catchpole and Feast, 1971). The SC group, with 12 stars in it, is particularly interesting. These stars are all very similar to one another spectroscopically except that one has a very strong lithium line (E.W. $\sim 3 \AA$ ). Infrared photometry (Glass, to be published) shows that the SC stars form a compact group in their $J, H, K, L$ colours. The infrared colours of the superlithium rich SC star place it with the other SC stars and appear to rule out the possibility that the great enhancement of lithium in this star is a temperature effect.

The mechanism to produce superlithium rich stars discussed by Cameron, Fowler and others is expected to operate in relatively massive stars ( $\sim 5$ solar masses or more) and not to be very effective at lower masses. With only 7 stars known in the superlithium rich group, it is not possible to deduce much on this point from their kinematics except that there are no very large (halo type) radial velocities amongst the seven. However, our new superlithium rich Se variable RZ Sgr has the relatively high velocity of $-38 \mathrm{~km} \mathrm{~s}^{-1}$ w.r.t.l.s.r. in a direction where the effects of differential galactic rotation are small and lies at a galactic latitude of $33^{\circ}$ and so is probably well out of the plane. Velocity and position thus suggest either a moderately old low mass object (contrary to theoretical expectation) or possibly a run away star.

In this paper some aspects of current work have been discussed for each of the three main abundance classes amongst red variables, the $\mathrm{M}, \mathrm{C}$ and $\mathrm{S}$ type stars. It will be apparent that in each group there are many unsolved problems requiring further observational and theoretical work. 


\section{Acknowledgements}

I am grateful to R. M. Catchpole, T. Lloyd Evans and P. J. Andrews for discussions and unpublished data.

\section{References}

Andrews, P. J.: 1973, Quart. J. Roy. Astron. Soc. 14, 451.

Bessell, M. S. and Youngbom, L.: 1972, Proc. Astron. Soc. Australia 2, 154.

Boesgaard, A. M.: 1970, Astrophys. J. 161, 1003.

Cameron, A. G. W. and Fowler, W. A.: 1971, Astrophys. J. 164, 111.

Catchpole, R. M. and Feast, M. W.: 1971, Monthly Notices Roy. Astron. Soc. 154, 197.

Catchpole, R. M. and Feast, M. W.: 1973, Monthly Notices Roy. Astron. Soc. 164, 11 P.

Demarque, P. and Mengel, J. G.: 1973, Astron. Astrophys. 22, 121

Dickens, R. J., Feast, M. W., and Lloyd Evans, T.: 1972, Monthly Notices Roy. Astron. Soc. 159, 337.

Dickens, R. J. and Powell, A. L. T.: 1973, Monthly Notices Roy. Astron. Soc. 161, 249.

Eggen, O. J.: 1972, Astrophys. J. 174, 45.

Feast, M. W.: 1953, Coll. Astrophys. Liège 5, 423 (Les Processus Nucléaires dans les Astres).

Feast, M. W.: 1963, Monthly Notices Roy. Astron. Soc. 125, 367.

Feast, M. W.: 1965, Observatory 85, 16.

Feast, M. W.: 1973, in J. D. Fernie (ed.), 'Variable Stars in Globular Cluster and Related Systems, Feast, M. W. and Lloyd Evans, T.: 1973, Monthly Notices Roy. Astron. Soc. 164, 15P.

Feast, M. W., Woolley, R. v.d. R., and Yilmaz, N.: 1972, Monthly Notices Roy. Astron. Soc. $158,23$. IAU Colloq. 21, 131.

Gascoigne, S. C. B.: 1966, Monthly Notices Roy. Astron. Soc. 134, 59.

Glass, I. S. and Feast, M. W.: 1973, Monthly Notices Roy. Astron. Soc. 163, 245 and 164, 423.

Johnson, H. L., Coleman, I., Mitchell, R. I., and Steinmetz, D. L. : 1968, Commun. Lunar Planetary Lab. $7,83$.

Keenan, P. C.: 1967, Astron. J. 72, 808.

Lloyd Evans, T., and Menzies, J. W.: 1973, in J. D. Fernie (ed.), 'Variables Stars in Globular Clusters and Related Systems', IAU Colloq. 21, 151.

Mendoza, E. E.: 1967, Bol. Obs. Tonantzintla Tacubaya 4, 114.

Preston, G. W.: 1967, Publ. Astron. Soc. Pacific 79, 125.

Rood, R. T.: 1972, Astrophys. J. 177, 681.

Truran, J. W. and Cameron, A. G. W.: 1971, Astrophys. Space Sci. 14, 214.

Warner, B. and Dean, C. A.: 1970, Publ. Astron. Soc. Pacific 82, 906.

Woolf, N. J., Schwarzschild, M., and Rose, W. K.: 1964, Astrophys. J. 140, 833.

\section{DISCUSSION}

Mavridis: I have two questions. First, did you determine the absolute magnitudes of the carbon stars in the Magellanic Cloud clusters? Second, can you tell us whether these stars are $M$ type or $F$ type stars?

Feast: The absolute magnitudes I can tell you. As to your second question, I would not like to say. Mavridis: Otherwise tell us whether they are early $\mathrm{C}$ or late $\mathrm{C}$.

Feast: I can tell you that, in these two clusters at any rate, if you determine the intensity of the carbon bands, these are about 3 on the Morgan and Keenan's scale (which runs from 0 to 10). Their absolute magnitudes are about -3 , which is somewhat brighter than the ones in the intermediate age galactic clusters in the galaxy, which are I think of the order of -2 .

Buscombe: The opacity, according to Hollis Johnson of Bloomington, Indiana, is more contributed by water vapour and by carbon monoxide than it is by TiO.

Feast: In the $5000 \AA$ region?

Buscombe: No, not there.

Feast: One wants to know what the opacity is in the region where you see the TiO in the spectrum.

Buscombe: He has models in which he imitates the observed spectra with computation based on opacity and he has been quite successful with it. 
Vardya: The $\mathrm{H}_{2} \mathrm{O}$ opacity operates around one micron. Thereafter the $\mathrm{CO}$ will also operate.

Feast: I do not know whether Dr Vardya would like to say whether it seems reasonable that TiO could provide a reasonable amount of opacity.

Vardya: It may. The thing is $\mathrm{H}^{-}$should go down and then the $\mathrm{TiO}$ can come up. That is very difficult to say.

Bell: I wanted to make one or two comments about the $\mathrm{CH}$ stars. Dickens and I have analysed the spectra of one of them in $\omega$ Centauri and we get a carbon 12 to carbon 13 ratio which is 10 instead of 90 in the solar case. We also get a higher nitrogen abundance than for the other elements which you expect to have an abundance of about $1 / 20$ of solar. The comment I wanted to make to start with, is that your diagram is a little bit misleading in that the $\mathrm{CH}$ stars are reddened by the carbon features in their spectra. They are in fact occurring at something like 0.1 or 0.2 mag. redder in $(B-V)$ than their temperature would indicate.

Feast: We got infrared observations of at least one of these and they do not lie anything like as far out as the $\mathbf{M}$ stars in $\omega$ Cen. They are somewhere in the region of the Giant Tip.

Bell: Another question I would like to ask you is: in the spectrum of your star in Kron 3, which is your 16th mag. star, can you see any evidence for the existence of carbon 13? Can you see the $\mathrm{Cl} 3-\mathrm{C} 13$ head at about $4754 \AA$ ?

Feast: I cannot tell you at the moment. I think one would have to work on the spectrum fairly carefully, which I have not done. It is a very interesting point.

Bell: Another point is related to the star which you are giving a $(B-V)$ of 2.4 . Such a $(B-V)$ could possibly be produced by excess carbon blanketing as well. Because if one can produce something like one or two tenths of magnitude in the $\omega \mathrm{Cen} \mathrm{CH}$ stars which have a relatively low $\mathrm{C} / \mathrm{O}$ ratio, then if you take a high $\mathrm{C} / \mathrm{O}$ ratio you could get very much greater carbon blanketing by $\mathrm{CN}$ and $\mathrm{CH}$ and consequent reddening of the star.

Feast: I am sorry, I certainly did not mean to imply that I thought the $(B-V)$ 's of carbon stars told you what the temperatures were. It is just of course that in the galactic globular clusters you can use the $(B-V)$ to see whether you find stars like this. Could I ask Dr Bell a question? In the analysis that you did with Bob Dickens, can you tell the difference between a carbon over-abundance and an oxygen underabundance?

Bell: It depends upon the temperature of the star because the strength of the $\mathrm{C}_{2}$ features is very strongly dependent upon the temperature. It seemed that, in $\omega$ Centauri for example, you could not quite produce strong enough $\mathrm{C}_{2}$ features if you took away all the oxygen. But of course the problem is that you do not really know what the oxygen abundance is.

One other question I would like to ask. Do you see any possibility of seeing carbon 13 features in your variable $\mathbf{M}$ stars. That could help you to decide whether or not they are on their first or second ascent of the giant branch.

Feast: Not in the spectra... If one went into the infrared it might be possible; they are not very bright stars, of course. It is a very interesting point.

Rodgers: Two points: one with respect to your comment about TiO supplying its own opacity. Ms Hain, now Mrs Shinkawa worked on S Carinae, and in working on field stars took $(R-I)$ observations and near infrared scans of continuum and band strengths in what she called low and high velocity giants. Professor Eggen made exactly the same observations and the results that come out of both of these are that the TiO band strengths are independent of what kinematics you assign to the star. On your picture it says either $\mathrm{TiO}$ is the dominant source of opacity or, for the high velocity stars, electrons for $\mathrm{H}^{-}$opacity are deficient. This is likely to be true for supergiants but for the giants, the $\mathrm{H}^{-}$opacity, is going down, and again you have this problem of if the $\mathrm{TiO}$ is down, what is the $\mathrm{O}$ doing.

The final point $I$ want to make is that even among the early $\mathbf{M}$ type stars where TiO is patently not supplying its own opacity there is still no differentiation. The second point - you made the point that in the Miras in $\omega$ Cen you had in $\log T_{\mathrm{e}}$ a red extension of the giant branch but no extention in luminosity. I would like to say that Dr Hyland and I are working on two Miras in NGC 6397, in which we think the metal abundance is down by at least 20 (this is the calcium abundance in the horizontal branch). These two stars lie two to three magnitudes below the giant branch in $V$, but in $M_{\text {bol }}$ are way above the tip of the giant branch of that cluster.

Feast: These are Miras?

Rodgers: Yes.

Feast: Quite large amplitudes?

Rodgers: Yes, in $V$. The light curves are not well determined. I give a minimum of about $4 / 10$ ths. 
Hyland: These are two stars which are right at the tip of the giant branch in NGC 6397 which on the $[V,(B-V)]$ diagram fall well below, as you said before. They were found to be variable by the Herstmonceux group and they did not give any period but you can get a rough sort of period between 100 and 200 days, and the large amplitude in $V$. In fact, when we first looked at one, it was 16th magnitude in $V$, in $\mathrm{K}$ it is third magnitude. So it is a pretty bright infrared source. Sixty days later, in $V$ it was about 11 th mag. Its $\mathrm{K}$ mag. had only changed by about $2 / 10$ ths mag. So this one is a really large amplitude in the optical but in the infrared it hardly varies at all, or has a very small amplitude. The other one is not as well determined as to what is going on.

The point about it, is that the two spectra of these stars with the TiO bands both show a spectrum around about M5. One of them is redder than the other and they have different calcium line strengths $(4226 \AA)$, which would indicate a difference in temperature. The one which is right up at the tip of the giant branch is much brighter than any in $\omega$ Cen or $47 \mathrm{Tuc}$; in fact one would expect it on its colour to have a spectral type of M7, M8... but it is still only M5 according to the TiO strength.

Przybylski: In addition to the comment made by Dr Rodgers I would like to stress one point concerning the TiO bands in late type stars in $\omega$ Centauri. Normally we would expect normal strength of TiO bands if oxygen is normal in the star. It is always so that if one component of a molecule has a normal abundance in the atmosphere then the strengths of the absorption bands does not change. That is what we observe, for instance, with $\mathrm{CH}$ bands. We all agree probably that in all kinds of stars, with a few exceptions only, hydrogen is normal and for this reason, the strength of $\mathrm{CH}$ bands is independent of the fact whether it is a star of population I or population II; exactly in the same way, for instance, $\mathrm{Mg} \mathrm{H}$ is also normal in population II stars. If oxygen is normal we would also expect that TiO bands would be normal. The possible conclusion may be that in $\omega$ Centauri, oxygen is normal. Does anyone know whether there is any observation and evidence to the contrary? Incidentally, what is the strength of CN bands in $\omega$ Centauri?

Feast: I do not think that I can give you a qualitative figure. I would be very interested to hear from the experts on stellar atmospheres whether they would agree with that comment.

Rodgers: Peimbert reported on the composition of planetary nebulae at the IAU Symposium No. 58. In the planetary in $\mathrm{M} 15$ where $\mathrm{Ca} / \mathrm{H}$ is down by thirty, oxygen is only down by a factor at 10 . It still seems to us very hard to understand the strong TiO in the NGC 6397 Miras because we would have thought that with these extremely low gravity stars, Rayleigh scattering must play a fairly major role. They are extremely cold and Rayleigh scattering, I would think, must be competing - we have not done the sums yet - very very strongly with intrinsic band absorption by $\mathrm{TiO}$. Of course, if you are right, we are underestimating the $\mathrm{TiO}$ abundance because the $\mathrm{TiO}$ band edges are in fact overlapping. It seems difficult to believe that these stars have the same surface composition as the rest of the stars in 6397 .

Feast: I am fairly confident that the $\mathbf{M}$ stars in $\omega$ Cen are metal-deficient objects. Of course, you can always fix up the strength of the $\mathrm{TiO}$ by varying the elements in an arbitrary way, but the fact that you have got to get back to the relation you find for normal abundance stars makes it rather suspicious to just juggle the abundances.

Vardya: I was wondering why some of the $\mathbf{M}$ stars become very much like Mira variables, others do not. Are there any clues from the observation side which might tell us what makes a star become Miras where others do not.

Feast: I did not have it on those slides but we have got some infrared observations of V42 in $\omega$ Cen which is one of the largest amplitude red variables. It has got a period of about 150 days and it may very well, if one uses the Mira terminology, be a metal deficient Mira. In the observations we have got in a $[(\mathrm{J}-\mathrm{H}),(\mathrm{H}-\mathrm{K})]$ diagram, it falls with the 47 Tuc Miras as though the opacity in the $\mathrm{H}$ region is being affected in this star in $\omega$ Cen, by water vapour. Of course, one has got to realise that these $M$ stars in $\omega$ Cen are pretty rare. A lot of stars were gone through to find that. 